\title{
The Long Noncoding RNA OIP5-AS1 Is Involved in the Regulation of Cell Proliferation
}

\author{
MADOKA NAEMURA ${ }^{1}$, MASAHIDE KUROKI ${ }^{2}$, TOSHIYUKI TSUNODA ${ }^{3,4}$, \\ NAGISA ARIKAWA ${ }^{1}$, YUUGA SAWATA ${ }^{1}$, SENJI SHIRASAWA ${ }^{3,4}$ and YOJIRO KOTAKE ${ }^{1,2}$ \\ ${ }^{1}$ Graduate School of Humanity-Oriented Science and Engineering, and \\ ${ }^{2}$ Department of Biological and Environmental Chemistry, \\ Faculty of Humanity-Oriented Science and Engineering, Kindai University, Iizuka, Japan; \\ ${ }^{3}$ Department of Cell Biology, Faculty of Medicine, and \\ ${ }^{4}$ Central Research Institute for Advanced Molecular Medicine, Fukuoka University, Fukuoka, Japan
}

\begin{abstract}
Background/Aim: OPA-interacting protein 5 antisense transcript 1 (OIP5-AS1) is a long noncoding RNA located on human chromosome 15q15.1 and transcribed in the opposite direction to OIP5. Here, we report that OIP5-AS1 is involved in regulating cell proliferation. Materials and Methods: HeLa cells were transfected with OIP5-AS1-targeting siRNA oligonucleotides and anti-sense oligonucleotides. The cells were harvested $72 \mathrm{~h}$ after transfection and subjected to quantitative reverse transcription-polymerase chain reaction (qRT-PCR), and cell-cycle and apoptosis analysis. Results: OIP5-AS1 was expressed at a lower level in cells harbouring an oncogenic kirsten rat sarcoma viral oncogene homolog $(K$ $R A S)$ mutation than in cells expressing wild-type K-RAS. Silencing OIP5-AS1 with siRNA oligonucleotides or anti-sense oligonucleotides reduced HeLa cell proliferation. Apoptosis and cell-cycle analysis showed that silencing OIP5-AS1 did not cause apoptosis, but did cause $G_{2} / M$ phase cell-cycle arrest. Conclusion: These results suggest that OIP5-AS1 positively regulates cell proliferation by promoting $G_{2} / M$ phase progression.
\end{abstract}

Long noncoding RNAs (lncRNAs) are longer than 200 nucleotides and lack functional open reading frames. IncRNAs form a heterogeneous group whose members

This article is freely accessible online.

Correspondence to: Yojiro Kotake, Ph.D., Department of Biological and Environmental Chemistry, Faculty of Humanity-Oriented Science and Engineering, Kindai University, 11-6 Kayanomori, Iizuka, Fukuoka 820-8555, Japan. Tel: +81 948225659 (Ext. 469), Fax: +81 94823053, e-mail: ykotake@ fuk.kindai.ac.jp

Key Words: Long noncoding RNA, OIP5-AS1, cell proliferation, cell cycle. exhibit different mechanisms of action and are involved in multiple cellular functions, including transcriptional regulation, nuclear structure organization, and posttranscriptional processing (1-3). Recently, we reported that expression levels of multiple lncRNAs fluctuate in response to exogenous and endogenous expression of an oncogenic form of the small GTPase Ras (called oncogenic Ras) (4). Among the lncRNAs whose levels were reduced by oncogenic Ras, antisense noncoding RNA in the INK4 locus (ANRIL) functions to promote cell proliferation (5-7). $A N R I L$ is located in the cyclin-dependent kinase inhibitor 2A (INK4) locus, which encodes three tumour-suppressor proteins: the cyclin-dependent kinase inhibitors p15 and p16, and alternative reading frame (ARF), which stabilizes the tumour-suppressor protein p53. We and Yap et al. have reported that ANRIL binds to and recruits polycomb repression complex $1 / 2$ to the INK4 locus, thus repressing p15 and p16 transcription $(5,6,8)$. Furthermore, we recently showed that $A N R I L$ promotes human colorectal cancer cell proliferation in both two- and three-dimensional culture (9). Increased levels of ANRIL have been observed in several human cancer types, such as gastric (10), prostate (6) and ovarian (11) cancer, which suggests that ANRIL is involved in oncogenic transformation.

OPA-interacting protein 5 antisense transcript 1 (OIP5AS1) is a lncRNA located on human chromosome $15 \mathrm{q} 15.1$ and evolutionarily conserved in vertebrates (12). Inhibiting cyrano, a zebrafish homolog of human OIP5-AS1, was shown to cause embryonic developmental defects (12). Recently, it was reported that silencing human OIP5-AS1 in HeLa cells promoted cell proliferation, which suggests that OIP5-AS1 functions to inhibit cell proliferation (13). OIP5AS1 binds to HuR and prevents it from binding to and stabilizing protein-coding mRNAs such as cyclin A2, thus inhibiting cell proliferation. In this study, we investigated the role of OIP5-AS1 in cell proliferation. 
A

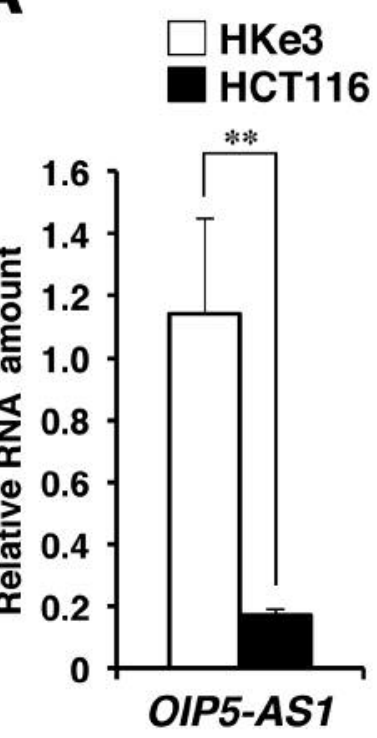

B

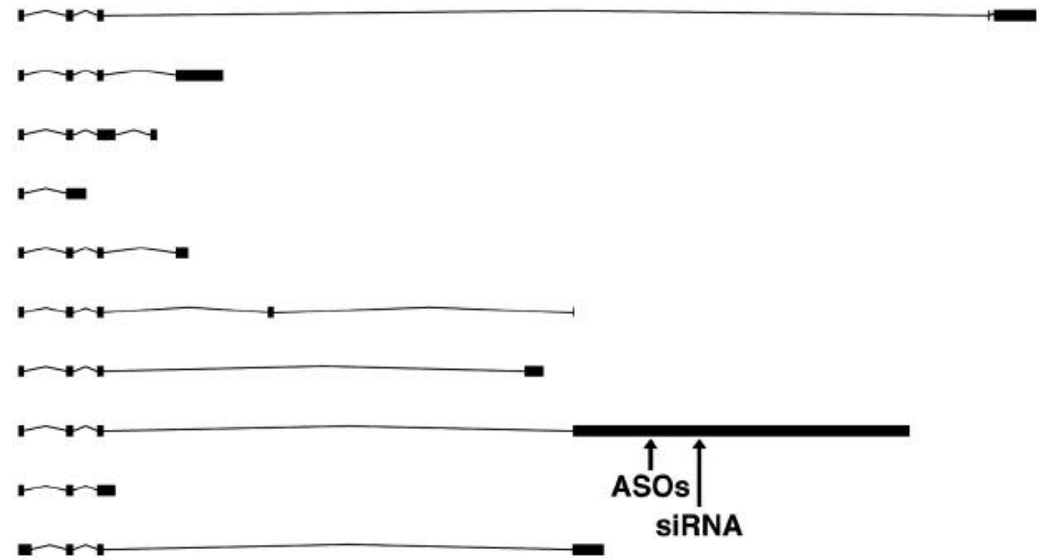

Figure 1. Opa-interacting protein 5 antisense transcript (OIP5-AS) expression and splice variants. A: OIP5-AS1 expression levels in HKe3 and HCT116 cells were determined by quantitative reverse transcription polymerase chain reaction. The results are expressed relative to the corresponding values for HKe3 cells. Data are presented as the mean \pm standard deviation ( $n=3)$. Data were analyzed using two-tailed Student's $t$ tests. **Significantly different at $p<0.01$. B: Schematic representation of OIP5-AS1 splice variants based on data from the Ensembl genome browser. Arrows indicate the positions of small interfering (si)RNA and anti-sense oligonucleotides (ASOs) designed for OIP5-AS1 silencing.

\section{Materials and Methods}

Cell culture. The HeLa human cervical cancer and HCT116 human colorectal cancer cell lines (American Type Culture Collection, Frederick, MD, USA) were grown in Dulbecco's modified Eagle's medium (Invitrogen, Carlsbad, CA, USA) supplemented with $10 \%$ foetal bovine serum (Gibco, Grand Island, NY, USA). HKe 3 cells were established from HCT116 cells with disrupted oncogenic K-RAS (14). The cells were cultured at $37^{\circ} \mathrm{C}$ in an atmosphere containing $5 \% \mathrm{CO}_{2}$.

Small interfering RNA (siRNA) and anti-sense oligonucleotide (ASO) transfection. siRNAs or ASOs were transfected into cells using Lipofectamine RNAiMAX (Invitrogen) or Lipofectamine 2000 (Invitrogen), respectively, according to the manufacturer's instructions. The nucleotide sequence of the OIP5-AS1-targeting siRNA was 5'-GCAGCAUGCUGUGUGCAAA-3' with 3' dTdT overhangs. The ASOs (Antisense LNA GapmeR) against OIP5-AS1 were produced by Exiqon (Vedbaek, Denmark). The ASOs contained locked nucleic acid and phosphorothioate modifications. The nucleotide sequence of the ASOs against OIP5-AS1 was 5'GAAGTTGGTAGATTAC-3'.

RNA extraction and quantitative reverse transcription-polymerase chain reaction ( $q R T-P C R)$. Total RNA was extracted using an RNeasy Plus kit (Qiagen, Hilden, Germany) according to the manufacturer's instructions. cDNA was synthesized using a SuperScript III FirstStrand Synthesis System (Invitrogen). q-PCR was performed using SYBR green PCR master mix (Qiagen) with specific primer sets as follows: human OIP5-AS1: 5'-TGCACATACACAGGTTAGA
ACAAG-3' and 5'-GAACCTAAACTTGGGTCTCTGG-3'; human glyceraldehyde-3-phosphate dehydrogenase $(G A P D H)$ : 5'-GCAAA TTCCATGGCACCGT-3' and 5'-TCGCCCCACTTGATTTTGG-3'.

Apoptosis analysis. Cells were labelled with CellEvent Caspase-3/7 Green Detection Reagent (Invitrogen) according to the manufacturer's instructions, then treated with Hoechst 33342 (Invitrogen). The fluorescence of cells was detected and analysed using an IN Cell Analyzer 2200 (GE Healthcare, Little Chalfont, UK).

Cell-cycle analysis. Cells were fixed in $70 \%$ ethanol overnight and stained using a Muse Cell Cycle Kit (Merck Millipore, Darmstadt, Germany) according to the manufacturer's instructions. The DNA content of the cells was analysed using a Muse Cell Analyzer and analysis software (Merck Millipore).

Statistical analysis. Data are presented as means and standard deviations. Statistical analyses were performed by two-tailed Student's $t$-test. $p$-Values of less than 0.05 were considered to be statistically significant.

\section{Results}

We previously reported that expression levels of multiple lncRNAs fluctuate in response to forced expression of oncogenic Ras (4). OIP5-AS1 is one lncRNA affected by oncogenic Ras signalling. We examined the levels of OIP5-AS1 in HCT116 and HKe3 cells. HCT116 cells harbour a 
A

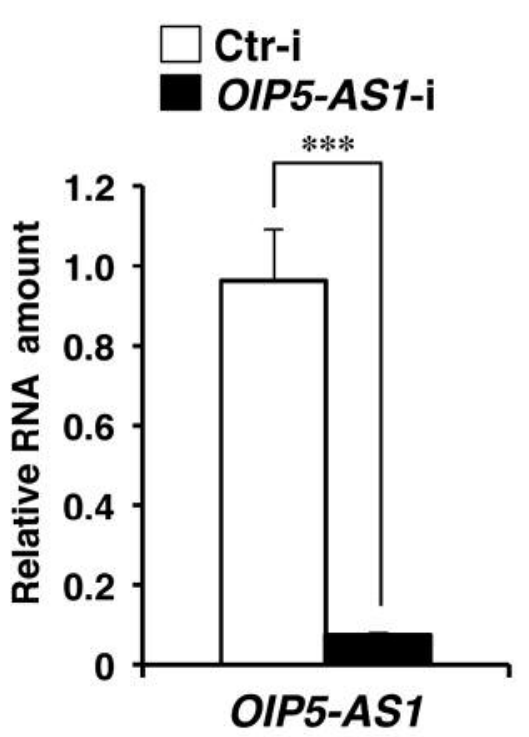

B

B

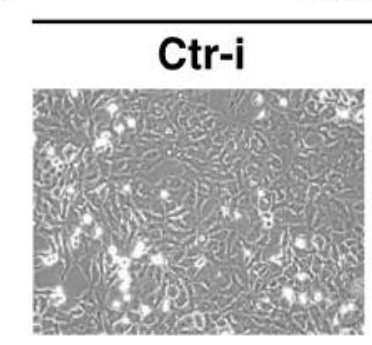

D

\section{$\square$ Ctr ASOs OIP5-AS1 ASOs}

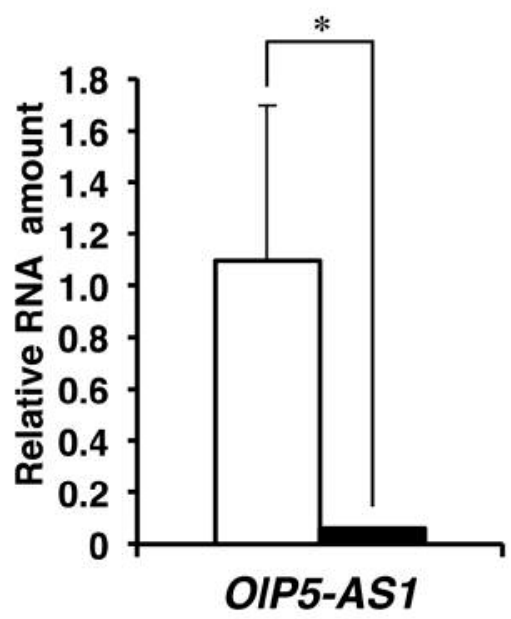

E

HeLa

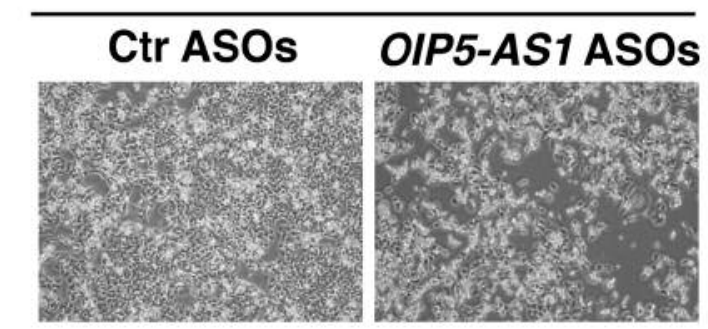

$\mathbf{F}$
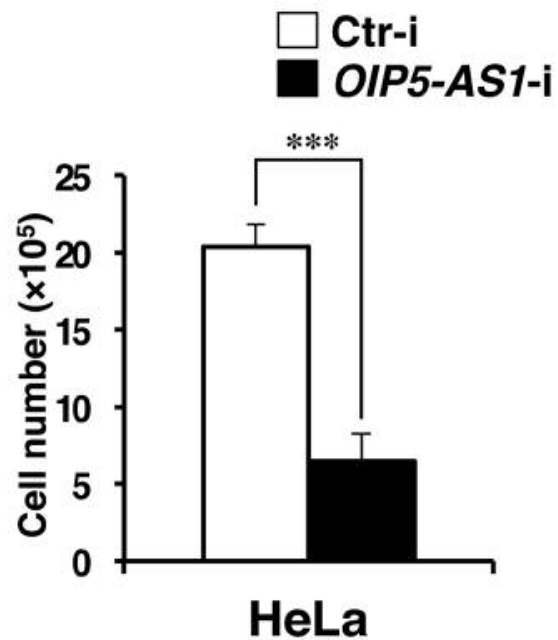

$\square$ Ctr ASOs

$\square$ OIP5-AS1 ASOs

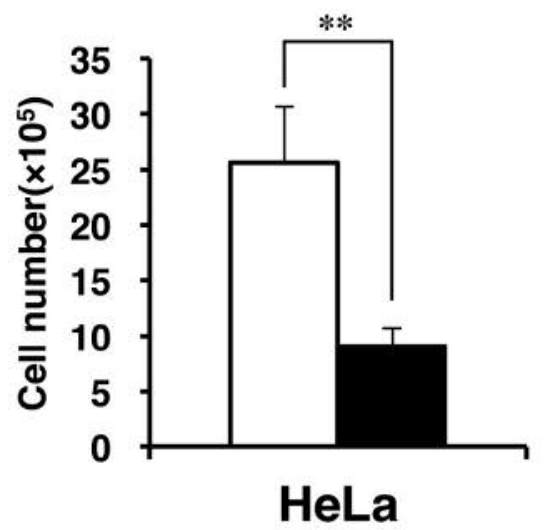

Figure 2. Depletion of opa-interacting protein 5 antisense transcript 1 (OIP5-AS1) by small interfering (si)RNAs or anti-sense oligonucleotides (ASOs) inhibits HeLa cell proliferation. A: HeLa cells $\left(2 \times 10^{5}\right)$ were seeded and incubated overnight, and then transfected with control (Ctr-i) and OIP5-AS1targeting (OIP5-AS1-i) siRNA. At $72 \mathrm{~h}$ after transfection, cells were harvested and subjected to quantitative reverse transcription PCR to determine OIP5-AS1 expression levels. The results are expressed relative to the corresponding values for HeLa cells transfected with control siRNA. B: HeLa cells transfected with siRNA were observed by phase-contrast microscopy at $72 \mathrm{~h}$ after transfection and counted by trypan blue staining (C). D: HeLa cells $\left(5 \times 10^{5}\right)$ were seeded and incubated overnight, and then transfected with control (Ctr) and OIP5-AS1-targeting ASOs. Quantitative reverse transcription $P C R$ was performed as in $(A)$. Imaging $(E)$ and counting $(F)$ of cells were performed as in $(B)$ and $(C)$. Data are presented as mean \pm standard deviation $(n=3)(A, C, D, F)$. Data were analysed using two-tailed Student's t-tests. Significantly different at: $* p<0.05$, ** $p<0.01, * * * p<0.001$. 
A
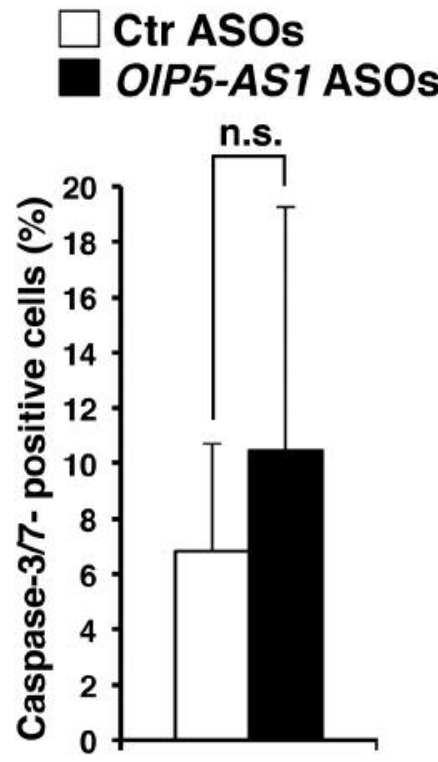

B
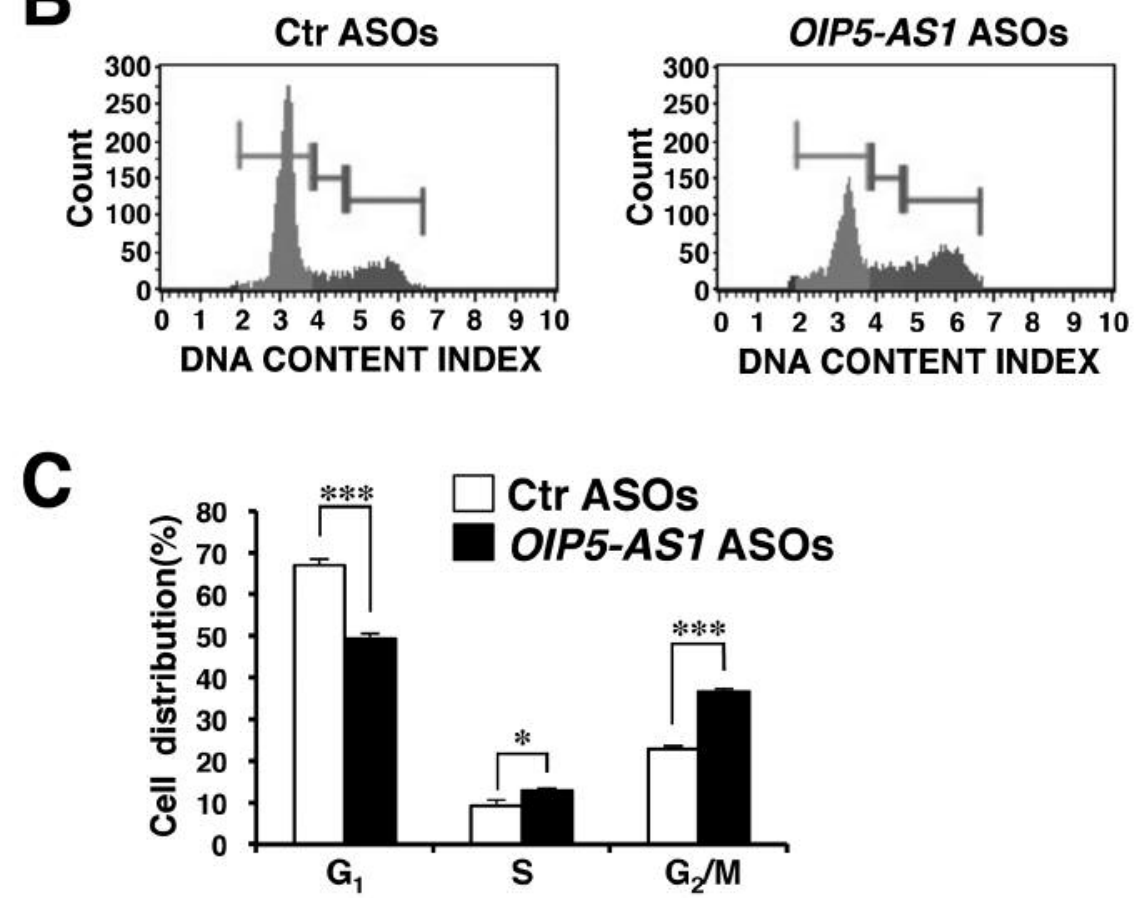

Figure 3. Silencing opa-interacting protein 5 antisense transcript 1 (OIP5-AS1) does not cause apoptosis, but does cause cell-cycle arrest in $G_{2} / M$ phase. A: At 72 h after transfection with anti-sense oligonucleotides (ASOs), cells were subjected to apoptosis analysis. The percentage of cells positive for active caspase-3 and -7 was determined by CellEvent Caspase-3/7 Green Detection. Data are presented as the mean standard deviation (n=3). B: At 72 h after transfection with ASOs, cells were harvested and subjected to cell cycle analysis. C: The percentage of cells in each cell cycle phase. Data are presented as the mean \pm standard deviation $(n=3)$. Data were analysed using two-tailed Student's $t$-tests. Significantly different at: $* p<0.05, * * * p<0.001 ;$ n.s., not significantly different.

heterozygous activating $K-R A S$ mutation (G13D); HKe3 cells were established by disrupting the mutated $K-R A S$ of HCT116 cells (14). qRT-PCR showed that OIP5-AS1 is expressed at a low level in HCT116 cells compared with HKe3 cells (Figure 1A), which indicates that oncogenic Ras signaling reduces OIP5-AS1 expression. We next elucidated the biological function of OIP5-AS1 in HeLa cells. OIP5-AS1 has several splicing variants (Figure 1B). We depleted expression of the major OIP5-AS1 transcriptional product using two different methods. We designed siRNAs for RNA interference-mediated degradation of OIP5-AS1 and ASOs for RNaseH-mediated degradation of OIP5-AS1 (Figure 1B). Transfection of siRNAs significantly reduced OIP5-AS1 expression (Figure 2A). Depleting OIP5-AS1 using siRNAs reduced the number of HeLa cells (Figure 2B and C). Depleting OIP5-AS1 using ASOs confirmed these results: transfection of ASOs, like siRNA transfection, reduced OIP5-ASI expression (Figure 2D) and repressed HeLa cell proliferation (Figure $2 \mathrm{E}$ and $\mathrm{F}$ ). These results suggest that $O I P 5-A S 1$ positively regulates HeLa cell proliferation. This is in contrast to the findings of a recent study by another group that showed that siRNA-mediated depletion of OIP5-AS1 promoted HeLa cell proliferation, which suggested that $O I P 5-A S 1$ functions to reduce cell proliferation (13). The reason for these conflicting results is not clear. The discrepancy might derive from the different target regions of the siRNAs or ASOs used to reduce the OIP5-AS1 level.

We next investigated the effects of OIP5-AS1 depletion on apoptosis and the cell cycle. OIP5-AS1 depletion did not affect the number of cells staining positively for caspase-3/7 activity (Figure 3A), which suggests that OIP5-AS1 is not involved in regulating apoptosis. Cell-cycle analysis demonstrated that OIP5-AS1 depletion reduced the proportion of cells in the $G_{1}$ phase and markedly increased the proportion of cells in the $\mathrm{G}_{2} / \mathrm{M}$ phase (Figure $3 \mathrm{~B}$ and $\mathrm{C}$ ). These results suggest that OIP5-AS1 positively regulates cell proliferation by promoting $\mathrm{G}_{2} / \mathrm{M}$ phase progression.

\section{Discussion}

In this study, we showed that OIP5-AS1 positively regulates HeLa cell proliferation. We showed that OIP5-AS1 is highly expressed in HCT116 cells harbouring an activating $K-R A S$ 
mutation compared to $\mathrm{HKe} 3$ cells established by disrupting the mutated $K-R A S$ of HCT116 cells (14), which suggests that $K$ $R A S$ signaling represses OIP5-AS1 expression. We previously reported that Ras signalling repressed the expression of $A N R I L$, a lncRNA that promotes cell proliferation by repressing the cyclin-dependent kinase inhibitor p15 (5). Ras signaling controls cell fate determination via processes such as cell proliferation, cell survival, apoptosis and cell-cycle arrest by regulating many downstream effector molecules (15). OIP5$A S 1$ and ANRIL may have an important role in determination of cell fate as downstream effectors of Ras signalling.

We demonstrated that depleting OIP5-AS1 using siRNAs or ASOs repressed HeLa cell proliferation, which supports the findings of a previous report that showed that inhibiting cyrano, a zebrafish homolog of human OIP5-AS1, causes embryonic developmental defects (12). Recently, another group reported that OIP5-AS1 functions to repress HeLa proliferation by binding to $\mathrm{Hu}$ Antigen $\mathrm{R}(\mathrm{HuR}$ ) and preventing it from binding to and stabilizing protein-coding mRNAs such as cyclin A2 (13). This is in contrast to our findings. This discrepancy may result from the different OIP5-AS1 regions targeted by the siRNAs or ASOs used to deplete OIP5-AS1. Supporting this, a recent study reported that the genes affected by the silencing of ANRIL exon 1 and 19 differ (16). Like OIP5-AS1, ANRIL has several splice variants (17). It might be that each splice variant of $A N R I L$ and OIP5-ASI has distinct target genes or functions.

Furthermore, we showed that depleting OIP5-AS1 caused cell-cycle arrest in $G_{2} / M$ phase. This finding suggests that OIP5-AS positively regulates cell proliferation by promoting $\mathrm{G}_{2} / \mathrm{M}$ phase progression. However, the molecular mechanism by which $O I P 5-A S 1$ promotes $\mathrm{G}_{2} / \mathrm{M}$ phase progression and the role of OIP5-AS1 in the Ras signalling pathway are yet to be determined and are important issues that require further investigation.

\section{Acknowledgements}

The Authors thank the members of the Kotake Laboratory for their technical assistance and helpful discussions. This work was supported by JSPS KAKENHI grant number 17K07184 (to YK) and the Naito Foundation (to YK). The Authors thank Ruth Tunn, Ph.D., from Edanz Group (www.edanzediting.com/ac) for editing a draft of this article.

\section{References}

1 Mercer TR, Dinger ME and Mattick JS: Long non-coding RNAs: insights into functions. Nat Rev Genet 10: 155-159, 2009.

2 Kitagawa M, Kotake Y and Ohhata T: Long non-coding RNAs involved in cancer development and cell fate determination. Curr Drug Targets 13: 1616-1621, 2012.

3 Wilusz JE, Sunwoo H and Spector DL: Long noncoding RNAs: functional surprises from the RNA world. Genes Dev 23: 14941504, 2009.
4 Kotake Y, Naemura M, Kitagawa K, Niida H, Tsunoda T, Shirasawa $\mathrm{S}$ and Kitagawa M: Oncogenic Ras influences the expression of multiple lncRNAs. Cytotechnology 68: 1591-1596, 2016.

5 Kotake Y, Nakagawa T, Kitagawa K, Suzuki S, Liu N, Kitagawa $\mathrm{M}$ and Xiong Y: Long non-coding RNA ANRIL is required for the PRC2 recruitment to and silencing of $p 15(I N K 4 B)$ tumorsuppressor gene. Oncogene 30: 1956-1962, 2011.

6 Yap KL, Li S, Munoz-Cabello AM, Raguz S, Zeng L, Mujtaba S, Gil J, Walsh MJ and Zhou MM: Molecular interplay of the noncoding RNA ANRIL and methylated histone $\mathrm{H} 3$ lysine 27 by polycomb CBX7 in transcriptional silencing of INK4a. Mol Cell 38: 662-674, 2010.

7 Naemura M, Murasaki C, Inoue Y, Okamoto H and Kotake Y: Long noncoding RNA ANRIL regulates proliferation of nonsmall cell lung cancer and cervical cancer cells. Anticancer Res 35: 5377-5382, 2015.

8 Kotake $\mathrm{Y}$, Naemura M, Murasaki C, Inoue $\mathrm{Y}$ and Okamoto $\mathrm{H}$ : Transcriptional regulation of the p16 tumor-suppressor gene. Anticancer Res 35: 4397-4401, 2015.

9 Naemura M, Tsunoda T, Inoue Y, Okamoto H, Shirasawa S and Kotake Y: ANRIL regulates the proliferation of human colorectal cancer cells in both two- and three-dimensional culture. Mol Cell Biochem 412: 141-146, 2016.

10 Zhang EB, Kong R, Yin DD, You LH, Sun M, Han L, Xu TP, Xia R, Yang JS, De $\mathrm{W}$ and Chen J: Long noncoding RNA $A N R I L$ indicates a poor prognosis of gastric cancer and promotes tumor growth by epigenetically silencing of miR-99a/miR-449a. Oncotarget 5: 2276-2292, 2014.

11 Qiu JJ, Wang Y, Liu YL, Zhang Y, Ding JX and Hua KQ: The long non-coding RNA ANRIL promotes proliferation and cell cycle progression and inhibits apoptosis and senescence in epithelial ovarian cancer. Oncotarget 7: 32478-32492, 2016.

12 Ulitsky I, Shkumatava A, Jan CH, Sive H and Bartel DP: Conserved function of lncRNAs in vertebrate embryonic development despite rapid sequence evolution. Cell 147: 1537-1550, 2011.

13 Kim J, Abdelmohsen K, Yang X, De S, Grammatikakis I, Noh JH and Gorospe M: LncRNA OIP5-AS1/cyrano sponges RNAbinding protein HuR. Nucleic Acids Res 44: 2378-2392, 2016.

14 Shirasawa S, Furuse M, Yokoyama N and Sasazuki T: Altered growth of human colon cancer cell lines disrupted at activated Ki-ras. Science 260: 85-88, 1993.

15 Karnoub AE and Weinberg RA: Ras oncogenes: split personalities. Nat Rev Mol Cell Biol 9: 517-531, 2008.

16 Congrains A, Kamide K, Katsuya T, Yasuda O, Oguro R, Yamamoto $\mathrm{K}$, Ohishi $\mathrm{M}$ and Rakugi H: CVD-associated non-coding RNA, ANRIL, modulates expression of atherogenic pathways in VSMC. Biochem Biophys Res Commun 419: 612-616, 2012.

17 Burd CE, Jeck WR, Liu Y, Sanoff HK, Wang Z and Sharpless NE: Expression of linear and novel circular forms of an INK4/ARFassociated non-coding RNA correlates with atherosclerosis risk. PLoS Genet 6: e1001233, 2010. 